\title{
Wavefunction reality, indeterminate properties and degrees of presence
}

\author{
Fedor Herbut \\ Serbian Academy of Sciences and Arts, Serbia, Belgrade, Knez Mihajlova 35
}

\begin{abstract}
The degree-of-presence (of the quantum system) concept, accompanying that of the wavefunction-reality postulate, is introduced and studied in two ways. To begin with, an incomplete exposition of the present author's views is given. Subsequently, a short historical and philosophical review of answers to the question about the meaning of indeterminate individual-system probabilities is presented from the literature. It is done in the form of a carefully selected collage of quotations mostly with polemic comments by the present author and with further elaboration of his point of view. The advocated notion of 'degree of presence' generalizes the intuitively most easily acceptable idea of 'delocalization' in (roughly called) wavelike behavior of a quantum system.
\end{abstract}

Keywords: Wavefunction reality; Individual-system probability; Propensity

\section{Introduction}

The assumption of a realistic interpretation of the wavefunction has a long standing. The origin of my personal interest for the subject lies in my recent research. A successful run of quantum-mechanical insights in intricate experiments assuming reality of the wavefunction of individual quantum systems has been performed by the present author (Herbut and Vujičić, 1997; Herbut 2008a; Herbut 2010a). Individual-system probability as an important open question has remained lingering on.

E-mail address: fedorh@sanu.ac.rs 
The last two mentioned studies of the present author deal with intricate variations on the two-slit interference experiment. As it is well-known, one has in its description a state vector (vector of norm one) $|\psi\rangle$ that consists of two components expressed via state vectors $|\psi\rangle_{i}, i=u, l$ as follows

$$
|\psi\rangle=\alpha|\psi\rangle^{u}+\beta|\psi\rangle^{l}, \quad \alpha, \beta \in \mathbf{C} \quad|\alpha|^{2}+|\beta|^{2}=1, \quad \alpha \neq 0 \neq \beta .
$$

The components mean passing the upper slit $u$ and the lower slit $l$ respectively, and they are orthogonal $\left\langle\left.\psi\right|^{u} \mid \psi\right\rangle^{l}=0$ due to disjoint respective domains $D^{u}$ and $D^{l}$ (around the slits) on which they are non-zero.

Further, the square moduli of the amplitudes $|\alpha|^{2}$ and $|\beta|^{2}$ are the probabilities that the particle can be found, in a so-called which-way measurement, to come from $D^{u}$ or $D^{l}$ respectively. One wonders how one should understand these probabilities having in mind the individual-system case.

One can find the following quotation on individual-system probabilities in a book of Pauli (1994, p. 43):

Quote Pa: "... incapable of further reduction ... a primary fundamental notion of physics."

Saunders (1998, p. 20) makes a similar remark:

Quote Sa: "Probability cannot be reduced to anything else: that is what I have been saying throughout."

Butterfield (2002) speaks of individual-system probability as follows:

Quote Bu: "[O]rthodoxy says: the probability distribution ascribed by the state is not for values possessed at some time or other; but for values yielded as result (pointer-reading) on a measurement apparatus. It is curious for the interpretation of state to invoke something so extrinsic to the system; and furthermore, for a typical measurement on a typical quantum system, something so vast compared with the system, so variable from occasion to occasion, and so vague. Indeed, it is surely more than curious: it is unsatisfactory, unless you have some general operationalist or instrumentalist view of theories." 
Butterfield speaks only of the manner how we, gross human beings, can take cognizance of the individual-system probabilities. The proposed 'degree of presence' concept is an attempt to grasp their true nature.

The term 'degree-of-reality' appears in the article by Busch and Jaeger (2010). Their idea differs from that of 'degree of presence' (see subsection 3.7 below for details). Sudbery (2011) gives a comprehensive discussion relevant for the individual-system probability concept (see subsection 3.8. below).

Since indeterminate properties can be viewed also as dispositions, the nice review article of M. Suárez (2007) is also worthy of reading. It reviews Margenau's latencies, Heisenberg's potentialities, Maxwell's propensitons, and the selective propensities of the author.

I feel that the question of the physical meaning of individual-system quantum probabilities deserves additional study.

In section 2 I'll introduce and explain the degree-of-presence concept. Section 3 is devoted to a collage of relevant quotes from other authors on indeterminate events or properties accompanied by a detailed comparison with the views of the present author, which are further elaborated where suitable.

In the collage of quotes, the first three authors (Aristotle, Reichenbach and Heisenberg) are mainly precursors of the degree-of-presence notion. The quoted views of the following five (Popper, Shimony, Mermin, Busch and Jaeger, and Sudbery) are inspirative for comparison and further elaboration of my standpoint.

\section{The present author's view of individual-system probabilities as degrees of presence}

As it is well known, quantum probabilities are measured on ensembles of equally prepared systems in the well-known frequentist way. One cannot doubt the reality of the ensembles, in particular, not the measurement results obtained in them. But then it is hard to imagine how one can doubt the reality of the states of their constituents - of the individual quantum systems that make up the ensemble.

The present author bases his position on this argument, and he is partial to the wavefunction-reality point of view. It is assumed throughout that the

wavefunction describes not only the corresponding ensemble, but also the 
state of the individual systems of which the ensemble consists.

\subsection{Gleason and degree of presence}

It is one of my interpretational postulates of the quantum formalism that the wavefunction and everything that it implies and is observable has reality, i. e., it exists 'out there' whether we observe it or not. The basic and most important implication of the wavefunction is probability: if $E$ is an event (or property or statement, mathematically a projector) and $|\Psi\rangle$ is a wavefunction (actually, a state vector, but we won't distinguish the abstract and the popular concrete representation form of this entity), then $\langle\Psi|E| \Psi\rangle$ is the probability of $E$ in $|\Psi\rangle$. We know, thanks to Gleason (1957), that also the converse is true, there is no other state, meaning the wider class of mixed-or-pure states, i. e., of density operators, that would give the same probabilities.

The way I understand this is that $|\Psi\rangle$ consists of these numbers as of a kind of its observable pieces when $E$ is running over the entire quantum logic $\mathcal{P}(\mathcal{H})$ (the set of all events - projectors - defined in the state space - Hilbert space - $\mathcal{H}$ of the system). In this manner, in the view of the present author, reality of $|\Psi\rangle$ logically implies that of $\langle\Psi|E| \Psi\rangle$. Since these are numbers from the doubly closed interval $[0,1]$, they can be called 'degrees of presence' of the quantum system in the corresponding elements of $\mathcal{P}(\mathcal{H})$. Since the projectors can, according to their physical meaning, be events or properties or quantum statements, one can also speak of degrees of presence of the quantum system in these quantum entities.

Naturally, one may view 'degree of presence' as a semantic variation of 'probability'. But making terms and endowing them with a physical meaning in the context of other physically meaningful notions (cf my comparison with Shimony's thoughts in subsection 3.5) seems to the present author to be the only way to learn to grasp quantum reality.

My understanding of 'degree of presence' is shaped on the concrete example of delocalization, where I find it easiest to imagine it. If standard quantum mechanics tells me that a particle passes coherently both slits in a two-slit interference experiment, and the probability of passing the upper slit is non-zero but small $\left(0<|\alpha|^{2} \ll|\beta|^{2}\right.$ in (1)), this does not mean, in my physical picture of the situation, that the passage of the upper slit is less real than that of the lower one; it means that the particle is in a lesser amount present there. This picture stems, of course, from the classical wave 
passing two openings simultaneously. For the time being I cannot do with fewer classical notions. (Incidentally, as to 'interference', cf Herbut (1992), and more can be read on 'coherence' in Herbut (2005a).)

The present author proposes to extend the interpretation of delocalization, i. e., of degrees of localization, to the spectrum of an arbitrary observable; particularly, to an arbitrary event or property or proposition and call it 'degree of presence'. More specifically, one may use the terminological variations degree of occurrence of an event, or degree of possession of a property, or degree of truth of a proposition respectively.

Perhaps it is not superfluous to stress again that, according to the advocated point of view, a lesser degree of presence does not mean less reality. Reality is all or none. It is none only if $\langle\Psi|E| \Psi\rangle=0$. A lesser degree of presence means that the system (the wavefunction of which we are concerned with) is to a lesser degree present in the entity in question (like in the case of delocalization).

A wavefunction is both reality and knowledge. A degree of presence that is smaller than 1 is not a partial or incomplete subjective information on a more complete quantum reality 'out there', but instead, it is complete information on an objective and complete form of a quantum reality.

One should note that here, like in relative-state Everett theory (Ecerett 1957 and Everett 1973), 'potential' or 'latent' is, by definition, banned from the interpretation. All parts, all components of the wavefunction, are real or actual (I use this term as a synonym for real), in general, but the system is in different degrees present in them.

Incidentally, I should point out that I strongly disagree with the still widespread view, originating with the main founder of quantum mechanics Niels Bohr (Petersen 1963) that the quantum-mechanical formalism has no other purpose than to provide us with numerical values for probabilities, which can then be tested experimentally. (Some 'rebels' against this prejudice call it "Shut up and calculate".) I believe that the quantum-mechanical formalism should teach us to comprehend quantum phenomena with as few prejudices from classical physics as possible. (Still, it might be considered a good thing that Bohr stopped large-scale inquiry into quantum insights, when he did it. It might have slowed down the rapid progress of the practical side of quantum mechanics that we have had up till now.) 


\subsection{Subsystems and reduced density operators}

One must keep in mind the fact that the physical systems of nature always come entangled. We know from the quantum formalism that this means that states of subsystems are usually described by reduced density operators that are more general than wavefunctions. The standard argument goes as follows:

$$
\left\langle\left.\Psi\right|_{12}\left(A_{1} \otimes I_{2}\right) \mid \Psi\right\rangle_{12}=\operatorname{tr}\left[\left(|\Psi\rangle_{12}\left\langle\left.\Psi\right|_{12}\right)\left(A_{1} \otimes I_{2}\right)\right]=\operatorname{tr}\left(\rho_{1} A_{1}\right),\right.
$$

where

$$
\rho_{1} \equiv \operatorname{tr}_{2}\left(|\Psi\rangle_{12}\left\langle\left.\Psi\right|_{12}\right)\right.
$$

(' $\operatorname{tr}_{2}$ ' denoting the trace over subsystem 2 ) is the state (reduced density operator) of subsystem 1 .

Hence, probabilities of subsystem observables (like of $A_{1}$ ) are actually predictions of the wavefunction of the larger system (of system $1+2$ in the notation of relation (2a)), and the concept of 'degree of presence' refers necessarily also to the reduced density operators, i. e., to improper mixtures (D’Espagnat 1976).

It should be pointed out that the 'degree of presence' concept does not apply to proper mixtures, which are density operators just like the improper ones, but physically they contain incomplete knowledge or ignorance. It was stated also by Heisenberg that proper mixtures lack full objectivity (cf subsection 3.3).

As a speculation, I think that one might use the term 'believed degree of presence' in the quantum Bayesian way (cf Caves et al. 2002) for the individual-system probability implied by a proper mixture.

\subsection{Entanglement and relative states}

There is a widespread dellusion that when one deals with a wavefunction $|\phi\rangle$ of a given physical system, then one may think of a world-wavefunction that factorizes tensorically

$$
|\phi\rangle|\Phi\rangle_{\text {rest-of-the-world }}
$$

For this to be valid the system under consideration would not be allowed to have interacted with any part of the rest of the world ever up till the present 
moment. This is so because entanglement is a kind of remnant of interaction, and it remains preserved in some form (cf Hulpke et al. 2006 and Herbut $2005 \mathrm{~b}$ ). Tensor factorization, on the other hand, means lack of entanglement.

One should call to mind that a wavefunction $|\phi\rangle$, as a rule, describes a system in a quantum experiment, and its state is obtained by preparation. As a consequence, the wavefunction is a conditional state in the collapse interpretation of quantum mechanics (cf my unpublished study Herbut (2010b)). It is a relative state in the no-collapse approach (Herbut 2011). I make use of the terms "approach" and "view" as of synonyms of " 1 ".

The advocated view of quantum mechanics was called 'relative reality of unitarily evolving state' (RRUES by acronym) in my recently published study Herbut (2008a). I am partial to the no-collapse or relative-state interpretation of quantum mechanics following Everett (1957 and 1973), which rests on 'unitary quantum mechanics', in which the Schrödinger equation - or equivalently the unitary evolution operator - rules all dynamics and collapse never happens. (More on 'unitary quantum mechanics' in my comments on some thoughts of Sudbery in subsection 3.8 .)

\subsection{Standard 'actualization' and occurrence}

Maximal degree of presence has a special position in standard quantum mechanics. It is interpreted as actualization, collapse, objectification etc., or specifically as occurrence of an event, possession of a property, truth of a quantum proposition. Let me present a first discussion of this notion as I see it.

To be concrete, I'll confine myself to events (projectors) $E$. If in a pure or improper mixed quantum state (density operator) $\rho$ an event satisfies $\operatorname{tr}(\rho E)=1 \quad$ (degree of presence equalling 1 ), then and only then one says in standard (collapse-interpretation) quantum mechanics that the event occurs in this state.

The basic role of the concept of 'degree of presence', or rather of 'degree of occurrence' in the case of events at issue, is, in my opinion, to put all positive values of individual-system probabilities on equal footing as far as reality is concerned. Occurrence takes place in different degrees.

Quantum events come in pairs $E, E^{c}$, where $E^{c} \equiv I-E$ ( $I$ being the identity operator) is the opposite event (the ortho-complementary 
projector). For all positive but non-maximal degrees of occurrence one has

$$
1>\operatorname{tr}(\rho E)>0<\operatorname{tr}\left(\rho E^{c}\right)<1 .
$$

A kind of 'quantum observer', if such existed, might take cognizance - via quantum correlations - of such a pair of events simultaneously. But classical physics knows only the extreme degrees of occurrence: an event occurs or it does not (then the opposite event occurs).

Observation of occurrence by human experimenters is performed with the help of classical apparatuses. Hence we, gross classical beings, must have actualization in the standard meaning, i. e., with maximal degree of presence.

But what is standard 'actualization' actually, one wonders. Of the many answers, let me just mention three: it is collapse of the wavefunction on the classical apparatus according to Bohr (and the Copenhagen interpretation Stapp(1972)); it is collapse in the consciousness of the sentient observer according to von Neumann (1955, last chapter); and finally, it is being in one of the branches or in one of the component universes of the multiverse according to the Everettian approach (Everett 1957, Everett 1973, De Witt 2004).

I am attracted to the Everett approach that guides my present investigations. But I have doubts (see my discussion in subsection 3.8 and the second concluding remark in section 4.).

\section{Review and comparison}

In this section a historical and philosophical selection of views on the notion of indeterminate properties predicted by a wavefunction is presented from the literature. The selection is, of course, incomplete and subjective. The review is displayed in the form of a collage of quotations accompanied by comments to find out, if possible, where the views of the reviewed author differ from those of the present author.

Incidentally, there are five terms in the literature (that I know of) designating positive probability less than 1 in application to individual quantum systems: "indeterminacy", "indefiniteness", "potentiality", "propensity" and "latency". 


\subsection{Aristotle}

In a remarkable recent article Sudbery (2011 pp. 8,9) writes:

Quote Su1: "Aristotle, in a famous passage (Aristotle 1980), considered the proposition "There will be a sea-battle tomorrow". He argued that this proposition is neither true nor false (otherwise we are forced into fatalism). Thus he rejected the law of excluded middle for future-tense statements, implying that they obey a many-valued logic. Modern logicians (Prior 1967) have considered the possibility of a third truth-value in addition to "true" or "false', namely u for "undetermined", for future-tense statements. But, interestingly, Aristotle admitted that the seabattle might be more or less likely to take place. This suggests that the additional truth values needed for future-tense statements are not limited to 1 , but can be any real number between 0 and 1 and should be identified with the probability that the statement will come true. Turning this round gives us an objective form of probability (italics by F.H.) which applies to future events, or to propositions in the future tense;..."

Aristotle couldn't, of course, imagine that one day some people would think, in principle, about everything, including the battle he was concerned with, in terms of a change of a wave function governed by the Schrödinger equation. But nevertheless, his thoughts might be considered to be an early, perhaps the earliest, precursors of the quantum-mechanical probabilities.

\subsection{The three-valued logic of Reichenbach}

Reichenbach (1965, paragraph 30), writing about how to characterize statements on latent or potential events, says:

Quote Re: "... This is achieved with the introduction of a third truth value of indeterminacy. The meaning of the term "indeterminate" must be carefully distinguished from the meaning of the term "unknown". The latter term applies even to two-valued

statements, since the truth value of a statement of ordinary logic can be unknown; we then know, however, that the statement is either true or false. The principle of the tertium non datur, or 
of the excluded middle, expressed in this assertion, is one of the pillars of traditional logic. If, on the other hand, we have a third truth value of indeterminacy, the tertium non datur is no longer a valid formula; there is a tertium, a middle value, represented by the logical status indeterminate."

Quantum mechanics does contain the "truth value indeterminacy", but, actually, one has more: something like a degree of indeterminacy, the subjective aspect of 'degree of presence' if the wavefunction is concerned.

Three-valued logic and probability goes back to Aristotle (1980). Important contributions are also in Lukasiewicz (1970), Reichenbach (1949), and Sudbery (2011).

\subsection{Heisenberg}

Heisenberg (1958, p. 53) wrote:

Quote He1: "The probability function combines objective and subjective elements. It contains statements about possibilities or better tendencies ("potentia" in Aristotelian philosophy), and those statements are completely objective, they do not depend on any observer; and it contains statements about our knowledge of the system, which of course are subjective in so far as they may be different for different observers. In ideal cases the subjective element in the probability function may be practically negligible as compared with the objective one. The physicists then speak of a "pure case." "

Predictions of wavefunctions are 'objective statements' in Heisenberg's wording. I cannot disagree with that. "Statements about our knowledge, that may be different for different observers" are implied by proper mixtures. They are much discussed lately (see Herbut 2004 and the references therein). They are almost completely excluded in this study because the 'degree-ofpresence' concept is not applicable to them.

The only point where Heisenberg apparently differs from the advocated degree-of-presence interpretation of probabilities implied by a wavefunction is that he seems to allow for a small subjectivity in the latter. This can be understood in two ways. Firstly, what he means may be that the wavefunction describes precisely and completely a model state of a model system, 
and this differs to some extent from the real state of the real system. The choice of such models may be due to the need to lessen complexity and to subjectivity.

Secondly, he might have been aware of the ubiquitous entanglement; Schrödinger (1935b and 1936) has brought them into focus. If a wavefunction is a conditional state (or a relative state, which is formally equivalent Herbut (2011)), it may be (formally) obtained from a proper mixture of a larger system containing ignorance. If any of these explanations is valid, Heisenberg's wording is acceptable to me.

Also Busch and Jaeger (2010, p. 20) point out that the notion of 'unsharp reality' began with Heisenberg (1959, p. 140), who claimed:

Quote He2: "... a course of events in itself is not determined by necessity but that the possibility or rather the 'tendency' towards a course of events possesses itself a kind of reality a certain intermediate level of reality midway between the massive reality of matter and the mental reality of an idea or picture..." ( translated from German by Busch and Jaeger).

Then the two authors add (Busch and Jaeger, ibid., p. 20). "He (meaning Heisenberg - F. H.) notes that this concept of possibility is given in quantum theory in the form of probability."

I have the impression that more than half a century ago Heisenberg, one of the great pioneers of quantum mechanics, was struggling both with the idea of "kinds of reality" and with the relation between "necessity" and randomness. The two dilemmas are absent from my RRUES approach (cf the third passage in 2.3). There is one kind of reality, the quantum reality given by the wavefunction, but, when particular events (or properties etc.) are concerned, it appears in different degrees of presence of the system, and they are observable in ensembles in the frequentist way. Necessity, i. e., determinism is universally valid in the multiverse. Randomness applies to the branches or particular worlds (more details in subsection 3.8).

I cannot help disagreeing with Heisenberg's words "a course of events in itself is not determined by necessity but that the possibility or rather the 'tendency' towards (italics by F. H.) ...'. The degree-of-presence notion has no dynamical side to it. In this respect Heisenberg is a precursor of Popper and his propensity (cf subsection 3.4.), not of the dynamically completely void 'degree-of-presence' concept.

Further Busch and Jaeger go on: 
Quote $(\mathbf{B}+\mathbf{J}) 1$ : "Heisenberg thus goes beyond the usual interpretation of the pure quantum state $|\psi\rangle$ as the catalogue of all actual properties - those with probability equal to one - of an individual system in that he considers $|\psi\rangle$ as the catalogue of the potentialities of all possible (sharp) properties Q of the system, quantified by the probabilities $\operatorname{psi}(Q)=\langle\psi|Q| \psi\rangle$ ".

In the terminology of Busch and Jaeger, 'sharp properties' are those represented by projectors, whereas the ones for which positive operators that are not projectors stand are 'unsharp properties'. The latter, if implied by a wavefunction, are always non-maximal degrees of presence.

I understand the words of Busch and Jaeger so that Heisenberg may have been the first author, or at least the first important author, who highlighted indeterminate properties of individual quantum systems.

\subsection{Popper's propensity}

Popper (1982, p. 159) wrote:

Quote Po1: "According to this picture...all properties of the physical world are dispositional, and the real state of a physical system, at any moment, may be conceived as the sum total of its dispositions - or its potentialities, or possibilities, or propensities."

These words sound as a precursor of Gleason's theorem (cf 2.1). Selleri and van der Merwe (1991, p. 1385), in the conclusion of their nice essay on Popper, write:

Quote S+vdM: "His proposal concerning propensities looks like an unescapable fundamental ingredient for any future realistic physics."

Popper's propensity is tendency for 'actualization', and the latter is 'occurrence' in the classical sense. Two more quotations make clear his position. On p. 351 (Popper 1983) he says:

Quote Po2: "[T] here is an analogy between the idea of propensities and that of forces - especially fields of forces. ... both ideas draw attention to unobservable dispositional properties of the physical world." 
Further, on p. 395 ibid. he states:

Quote Po3: "I propose to interpret the objective probability of a single event as a measure of an objective propensity - of the strength of the tendency, inherent in the specified physical situation , to realize the event - to make it happen."

Thus, Popper's 'propensity' is the tendency for standard actualization, and even with some dynamical attributes. This is the reason why I cannot accept it (and I cannot agree with Selleri and van der Merwe). I have begun to explain my view of actualization in subsection 2.4, and I'll give further elaboration in my comments on some ideas of Shimony in subsection 3.5.

Thinking along lines that Popper initiated has moved on (Suárez 2004 and others) apparently with more mathematical and philosophical sophistication. But, it seems to the present author, that the reality-of-wavefunction point of view requires a simpler idea for the indisputably primitive concept of individual-system probability.

\subsection{Shimony}

Shimony (1993, second passage p. 179), in the context of Einstein's world-view, discusses indefinite quantum properties as follows.

Quote Sh1: "The first of Einstein's theses ...., and the one which appears to stand highest in his philosophical hierarchy, is that physical things "claim a 'real existence' independent of the perceiving subject." This thesis is consistent with all the conclusions which we drew from an analysis of Bell's theorem, the relevant experiments, and the formalism of quantum mechanics. However, this thesis of physical realism, when separated from the rest of Einstein's theses, leaves open the character of the real existence of physical things. The foregoing analysis led to radical conclusions regarding the character of physical existence: i. e., that there are objective indefiniteness, objective chance, and objective probability (italics by F.H.), in short, that there is a modality of existence which has been designated as potentiality. ..."

One can find additional elucidation of his ideas in the following text (ibid., p. 142, 2nd passage): 
Quote Sh2: "The combination of indefiniteness of value with definite probabilities of possible outcomes can be completely referred to as potentiality, a term initially suggested by Heisenberg (1958, p. 185). When a physical variable which initially is only potential acquires a definite value, it can be said to be actualized. So far, the only processes we have mentioned in which potentialities are actualized are measurements, but in a nonanthropocentric view of physical theory the measurement process is only a special case of the interaction of systems, of special interest to scientists because knowledge is thereby obtained, but not fundamental from the standpoint of physical reality itself...."

Shimony's clear and illuminating text highlights the concept of 'actualization' in an inspirative, but standard way. He mentions measurement, but does not (at least not in the above excerpts) really point to the great problem connected with measurement. Namely, "in a non-anthropocentric view of physical theory the measurement process is only a special case of the interaction of systems" - he says. But in a collapse interpretation of quantum mechanics this is not so because 'collapse' is not a 'special case' of dynamical evolution (cf von Neumann 1955, p. 351). This is known as the 'measurement paradox'.

It is a problem only in collapse is. In a no-collapse (relative-state) approach measurement does not constitute a problem. There well defined measurement interaction Busch et al. (1996) leads to a so-called premeasurement state of object plus measuring instrument, in which the measurement results have been copied to the measuring instrument state. There is no problem except that the theory leads to the parallel worlds about which I'll say more in my comments on some ideas of Sudbery (cf subsection 3.8).

Another follower of Einstein, Bell found the Everettian no-callapse approach "extravagant" (on account of the parallel universes, no doubt). Therefore, he tried to comprehend 'measurement' within collapse quantum mechanics. He wrote an article (Bell 1990) under the provocative title "Against Measurement". This led to a beautiful controversy.

Reaction came from Peierls (1991) and from Gottfried (1991). The latter's article had the title "Does Quantum Mechanics Carry the Seeds of Its Own Destruction?". (My answer would be: Not quantum mechanics, but perhaps its collapse interpretation.) Gottfried's reaction was criticized by Whitaker (2008). Also Mermin (2006) reacted to Bell's criticism of measurement. His 
argument, in its turn, was critically examined by Ghirardi (2008).

The difficulties with the 'measurement' concept in collapse quantum mechanics are a strong indication that one should take the Everettian approach into serious consideration. But one might retort that one thus barters one burden for another, perhaps ending up with a more serious one. One must find out which 'burden' is the lighter one, which can be disposed of. The measurement problem is with us for almost a century.

Measurement is performing the 'actualization' that Shimony is concerned with. I'll present now a short exposition of how I see the connection between the three key concepts, entanglement, degree of presence, and actualization.

Let $|\Psi\rangle_{12}$ be an arbitrary state vector of a composite system (a socalled bipartite state). It always has a bi-orthogonal expansion with real coefficients (so-called canonical Schmidt expansion, cf subsections $2.1-2.3$ in Herbut (2007) for a review)

$$
|\Psi\rangle_{12}=\sum_{i} r_{i}|i\rangle_{1}|i\rangle_{2}, \quad \forall i, i^{\prime}: \quad\left\langle\left. i\right|_{1} \mid i^{\prime}\right\rangle_{1}=\left\langle\left. i\right|_{2} \mid i^{\prime}\right\rangle_{2}=\delta_{i, i^{\prime}}
$$

The sum is finite or infinite; the number of terms is called the Schmidt rank. It is uniquely determined by the bipartite state, and so are the expansion coefficients $\left\{r_{i}: \forall i\right\}$, though the basis $\left\{|i\rangle_{1}: \forall i\right\}$ in expansion (3) is not. A good quantitative measure of the amount of entanglement in $|\Psi\rangle_{12}$ is the so-called mutual information (cf Herbut 2005c) $I \equiv-\sum_{i} r_{i} l n r_{i}$.

Let the Schmidt rank, say $N$, be, for simplicity, finite $1<N<\infty$. Then the maximal value of the mutual information is $I_{\max }=\ln N$, and $r_{i}=1 / N, i=1,2, \ldots, N$. Thus, for large $N$, we obtain a large amount of entanglement if the degrees of presence of the events $|i\rangle_{1}\left\langle\left. i\right|_{1} \text { in } \mid \Psi\right\rangle_{12}$, $r_{i}$, are very small. Conversely, if some of the degrees of presence $r_{i}$ are large, the mutual information is small. In the opposite extreme case, when there is no entanglement $(\mathrm{N}=1)$, the degree of presence is 1 .

In this sense the amount of entanglement and the degree of presence of suitable subsystem events, 'correlations' and 'correlata' (see the next subsection), are kind of complementary to each other.

The opposite-subsystem events $|i\rangle_{1}\left\langle\left. i\right|_{1} \text { and } \mid i\right\rangle_{2}\left\langle\left. i\right|_{2}\right.$ in relation (3) not only have the same degree of presence $r_{i}$, but they are also the relative states with respect to each other (conditional states of each other in collapse 1). One can say that they carry information about each other. 
Viewing things in the way I have just suggested, there is nothing special about 'actualization', when $N=1$. Loosely speaking, quantum systems can 'know about one another' for any non-zero degree of presence. We need 'actualization' in the standard sense because we are incarcerated in a classical world, where the degrees of presence of events are confined to zero and one. If we want to understand the quantum world, we must consider all possible quantum values of the degrees of presence on the same footing. In other words, we should understand 'actualization' as a synonym of 'reality'. It is then valid for all positive degrees of presence.

\subsection{Mermin}

Mermin (1998) writes (in section III with the title "The puzzle of objective probability"):

Quote Me1: "[T]he fundamental role probability plays in quantum mechanics has nothing to do with ignorance (unlike in classical physics - remark by the present author). The correlata those properties we would be ignorant of - have no physical reality. There is nothing for us to be ignorant of.... We lack an adequate understanding of how probability or correlation is to be understood ... throughout this essay I shall treat correlation and probability as primitive concepts..."

"The correlations not the correlata" has become a well-known mantra of Mermin's "Ithaca interpretation" program, which I consider to be very promising and, above all, inspiring. A multipartite wavefunction usually contains entanglement, the most quantum kind of correlation; it is real and it represents a significant part of the reality of the wavefunction.

To illustrate the important notion of correlata, let me upgrade the above two-slit experiment (cf the Introduction) into the one in the real experiment of Kim et al. (2000), where instead of slits one has two excited atoms that emit in superposition, i. e., coherently, by cascade de-excitation a pair of photons (in opposite directions):

$$
|\Psi\rangle_{12}=(1 / 2)^{1 / 2}\left(|\psi\rangle_{1}^{u}|\psi\rangle_{2}^{u}+|\psi\rangle_{1}^{l}|\psi\rangle_{2}^{l}\right)
$$

where $i=1,2$ label the two photons, and $j=u, l$ refer to the two atoms that have emitted the two photons (counterparts of the upper and the lower slit). 
The subsystem events (projectors) $|\psi\rangle_{1}^{u}\left\langle\left.\psi\right|_{1} ^{u} \text { and } \mid \psi\right\rangle_{2}^{u}\left\langle\left.\psi\right|_{2} ^{u}\right.$ of the opposite photons, cascade partners meaning emission from the 'upper' atom, are correlated in $|\Psi\rangle_{12}$ : if photon 1 is emitted from the atom $u$, then so is photon 2 due to the cascade de-excitation. They, and symmetrically when one replaces $u$ by $l$, are the correlata. Mermin is right claiming that they "have no physical reality" if, in keeping with the standard view, one restricts oneself to maximal degree of presence or, speaking in the concrete terms of events, when one defines as occurrence only probability-one occurrence as in classical physics and in the Copenhagen interpretation of quantum mechanics (see Stapp 1972).

In the approach supported by the present author one takes into account all possible degrees of presence from zero to one (in particular, all degrees of occurrence in the open interval $(0,1))$. Therefore, in the view of the present author, the above correlata do have reality, but their degree of presence, i. e., the degree of the systems presence in the correlata is less than 1 ; in particular, it is $1 / 2$.

Let me repeat that, to my mind, a lesser degree of presence does not mean less reality. Reality is all or none. A lesser degree means that the quantum system is 'there' in a smaller amount (like in delocalization).

Thus, Mermin and myself seem to share the belief in reality of the individualsystem probabilities; though he, unlike myself, may not extend this to all probabilities. But we certainly differ in our views on the correlata. Mermin's words "There is nothing for us to be ignorant of" are not valid in this approach.

Perhaps Mermin's and my views on reality differ more than one would suspect at first sight. Mermin determines the concept of correlations in terms of mean values of all observables (Hermitian operators) of the subsystems that are correlated (Mermin 1998, p. 754)) including, of course, projectors. He attributes reality to the correlations, and reality of the probabilities seems to be a consequence as far as they partake in correlations. Then, probabilities implied by improper mixtures might not be real for Mermin, though they are real for me (cf subsection 2.2).

In a preliminary study (Herbut 2008b, not published yet) it was shown that, following Mermin, one can restrict the observables used by Mermin to events, and ipso facto determine the correlations in terms of probabilities only. (One can essentially further restrict even the set of events utilized.) In this manner, one can derive the reality of correlations from that of 


\section{probabilities.}

Let me put this clearly: In my view, on ground of Gleason's theorem, the individual-system probabilities derive their reality directly from that stipulated for the wavefunction (cf 2.1). Correlations derive theirs from that of the probabilities, and so do the correlata if their probability is positive.

But, of course, I may have misunderstood Mermin. If he believes in the reality of the wave function, then it seems unlikely that he can doubt the reality of any observable part of it.

Perhaps surprisingly, Mermin and myself seem to agree up to a point in utilizing the no-collapse relative-state approach to understand actualization. He says (on p. 755) of Mermin (1998):

Quote Me2"To the extent that "I" am describable by physics, which deals only with the correlations between me and the photomultipliers (phm), physics can only (correctly) assert that photomultiplier phm-n firing is perfectly correlated with my knowing that photomultiplier phm-n fired for either value of $n$. The question that physics does not answer is how it can be that I know that it is phm-1 and is not phm-2. This is indeed a problem. It is part of the problem of consciousness."

Mermin's formulation "to the extent that "I" am describable by physics" shows reservation towards the no-collapse approach. I do not share this reservation. But still he allows the approach, and I agree with him about the perfect correlations. Instead of "I" in his text, one could have a classical measuring apparatus. Then, "to the extent that" quantum mechanics is extended to classical systems, in particular detectors, one has the famous paradox of measurement in quantum mechanics. If, on the other hand, Mermin's "I" is replaced by "my friend", then we have the known paradox of Wigner 's friend (Wigner 1961).

An Everettian no-collapse approach, the point of view that is taken by me, treats all these cases on the same footing (see subsection 3.8). This approach is called the 'relative-state' one precisely because every wavefunction is taken in relation to a well-defined subsystem and pure state of it (in Everett's original expositions) or any event on the subsystem (in my straightforward elaboration of Everett, Herbut 2011). This subsystem plus an event on it is a subject entity. It is a premise, in my view, of Mermin's text (or of an analogous text with the measuring apparatus or with Wigner's friend). 
When Mermin is aware of seeing phm-1 and not any other value of $n$, one has a different subject entity: Mermin plus the event 'he is aware of phm1 '. In the relative-state approach this is not a "problem of consciousness" (or a problem of a classical measuring apparatus, or of Wigner's friend). It is just a consistent use of the quantum-mechanical formalism. (It need not be put aside as in Mermin's presentation.)

What has been said should, of course, be distinguished from the problems like 'what is conscousness', 'what are classical systems', 'what is Wigner's friend when Wigner has not asked him yet what result he sees'. For me all "photomultiplier phm-n firings" are realities, but relative to different subject entities. This is why I call my understanding of the no-collapse relative-state approach 'relative reality of unitarily evolving states (RRUES)' (cf 2.3) as in my previous study Herbut (2008a).

\subsection{Busch and Jaeger}

Busch and Jaeger (2010 subsection 2.4 there) write:

Quote $(\mathbf{B}+\mathbf{J}) 2$ : "We noted above that what is actual has the power to act. Similarly, when a property is absent there is no power to act. If this idea is extended to apply to the intermediate mode of existence, potentiality, one may say that an indeterminate property has a quantifiable, limited degree of reality (italics by F. H.) that manifests itself in a limited capacity potentiality to induce the associated measurement outcome. A quantitative measure of the degree of reality and associated potentiality is given by the quantum mechanical probability, which provides the likelihood for an individual outcome to occur in the event of measurement."

The authors of this excerpt use the term "degree of reality". For them the indefinite properties of individual-system wavefunctions are not on the same footing as the definite properties (maximal degree of presence). It is claimed that the former have limited reality, i. e., less than full reality, if I understand it correctly. Besides, I cannot agree with the dynamical ideas ascribed to indefinite properties like "power to act" or lack of it.

They write "limited capacity potentiality to induce the associated measurement outcome". From my point of view they fail to make a distinction between more inherent attributes of the state of the individual system, such 
as the degrees of presence in case of a wavefunction, and the more external ones that come to the fore when we observe them (in the frequentist way, cf the first concluding remark in section 4.). But even the latter are, in my opinion, without any "power to act". It appears that Busch and Jaeger are under the influence of Popper's propensity, which I have criticized above.

Actualization comes about in measurement, when the quantum world meets the classical one, and degrees of presence become converted, via ensembles, into frequencies. Raising actualization to an exalted position in comparison with the degrees of presence that are less that 1 when considering more inherent properties of the state of the individual system may result in a failure to grasp quantum essentials.

Another interesting passage from the article of Busch and Jaeger (ibid. on p. 2) reads:

Quote $(\mathbf{B}+\mathbf{J}) 3$ : "On a realist interpretation of Quantum Mechanics as a complete theory, the referent of quantum mechanical propositions is the individual system. This would not only recognize the possibility that such a philosophically realist interpretation could in the end enable the best description of the physical world; it also has the potential benefit of providing us with guidance in developing new, appropriately adapted intuitions about microphysical objects."

This is a good answer to the possible objection to the advocated approach claiming that degree-of-presence is just another term for probability. It is more than that. It means putting a concept, individual-system probability, in its proper place, in its proper conceptual context. This may pave the way for a deeper understanding. 


\subsection{Sudbery}

Now I present Sudbery's version of, what is called, the many-worlds or the multiverse view of universally valid quantum mechanics. Subsequently, I'll give my comments that will make clear to what extent I do agree with him.

On page 4 Sudbery (2011) one can read:

Quote Su2: "External truth: The truth about the universe is given by a state vector $|\Psi\rangle_{U}$ in a Hilbert space $\mathcal{H}_{U} \quad$ evolving according to the Schrödinger equation. If the Hilbert space can be factorised as $\mathcal{H}_{U}=\mathcal{H}_{S} \otimes \mathcal{H}_{E}$, where $\mathcal{H}_{S}$ contains states of an experiencing observer, then

$$
|\Psi(t)\rangle=\sum_{n}\left|\eta_{n}\right\rangle\left|\Phi_{n}(t)\right\rangle
$$

and all the states $\left|\eta_{n}\right\rangle$ for which $\left|\Phi_{n}(t)\right\rangle \neq 0$ describe experiences which actually occur at time t. Internal truth from the perspective $\left|\eta_{n}\right\rangle$ : I actually have experience $\eta_{n}$ which tells me that the rest of the universe is in the state $\left|\Phi_{n}(t)\right\rangle$. This is an objective fact; everybody I have talked to agrees with me."

Essentially, we are back at the problem of Mermin (cf quote "Me2"), of Wigner's friend, of the measurement paradox etc.

To begin with, I would prefer to include in $\left|\eta_{n}\right\rangle$ (one possible version of) the entire classical world with all possible active or passive observers in it. Sudbery's insistence on subjective "experience" might go under the name 'many-minds' instead of 'many worlds' as done occasionally. I do not think that there is any important difference.

Sudbery does not mention decoherence, which has been introduced implicitly by Zeh (1970) as the dynamical source for the expansion (5) (cf also Joos et al. (2003), especially Zeh's article there). Further, the terms in (5) are then considered as the parallel worlds.

The lhs of (5), representing the "external truth", is the state of the multiverse. It changes in a deterministic fashion according to the Schrödinger equation, and, I agree with Sudbery, that it is devoid of probabilities. But it is devoid of other attributes as well (see my 'doubt' below). The probabilities appear in the "internal truth". 
What I find disturbing is the fact that my own person (as well as yours, dear reader) is included in $\left|\eta_{n}\right\rangle$, and, in Sudbery's internal view, this is the case for just one value of $n$. But in Sudbery's external view there are many values (actually infinitely many) of $n$ all include me and all living creatures. This makes us a component not a subsystem (like the infamous cat of Schrödinger (1935a), which appears as a live and a dead component). The subsystem is given by $\mathcal{H}_{S}$ and it comprises all the components.

If this is all true, an ancestor of mine, when technology advances sufficiently, may, like in television stories, 'meet' or rather become one, with another component of himself.

Disturbing or not, if one assumes universal validity of the quantum formalism, it leads to decomposition (5).

I am personally prepared to go along with the expounded Everettian universally-valid quantum-mechanical vision of the multiverse all the way up to relation (5). But here I have a serious doubt.

Aware of a post-Everett standpoint of Wheeler (1977, p. 2), the very mentor and promoter of Everett's theory in the beginning (cf Byrne 2010 and Wheeler 1957), who, in turn, followed Wigner (1973, pp. 382-383), I share their opinion that the lhs of (5) has no physical meaning.

The wave functions $\left\{\left|\Phi_{n}(t)\right\rangle: \forall n\right\} \quad$ (cf (5)) from Sudbery's internal view are, in my opinion, legitimate extrapolations from our quantum experiments in the physical laboratory, but the lhs of (5), i. e., the wavefunction $|\Psi(t)\rangle_{U}$ of the multiverse ('universe' in Sudbery's terminology), is not. There is no external observer because $U$ is by definition everything. Then observables, and measurement, and all the concepts of which quantum mechanics consists are not valid for this wavefunction. One obtains it as a "view from nowhere" (as Sudbery mentions). I think that no-collapseoriented foundationally-minded physicists and philosophers are still wrestling with the idea of parallel universes (cf Saunders et al. 2010 and Carr 2007).

One might consider $|\Psi\rangle_{U}$, the lhs of in (5), as deprived of physical meaning, but still as a valid mathematical 'envelope concept' or 'umbrella notion' to encompass all possible proper subsystem states, which have full physical meaning.

For the reason of analogy, let me take resort to special relativity theory. The Minkowski space has itself no immediate physical meaning, but every 
one of its elements, points, is a possible event with full physical meaning. The space is just a mathematical 'envelope concept' enabling us to view all relevant possibilities.

Incidentally, in the Everettian expansion (5) $\left|\Phi_{n}(t)\right\rangle$ is a relative state taken relative to the subject state $\left|\eta_{n}\right\rangle$. In the language of textbook quantum mechanics the former would be called a conditional state, that would come about if the condition, the event $\left|\eta_{n}\right\rangle\left\langle\eta_{n}\right|$, occurred giving rise to the collapse of the lhs of (5) into one of the terms $\left|\eta_{n}\right\rangle\left|\Phi_{n}(t)\right\rangle$ on the rhs.

Another important passage in Sudbery's article argues against a Bayesian (purely subjectivistic, Caves et al. 2002) interpretation of probability. It reads as follows ( ibid. p. 8).

Quote Su3: "... our experience in a quantum-mechanical world seems to require a description in terms of objective chance. Things happen randomly, but with definite probabilities that cannot be reduced to our beliefs. The value of the half-life of uranium 238 is a fact about the world, not a mere consequence of someones belief."

The present author agrees with Sudbery completely concerning purely subjective individual-system quantum probabilities.

\subsection{Einstein's boxes and delocalization}

Let me first mention the famous Einstein boxes (Norsen 2005; sometimes called De Broglie boxes). A wavefunction $|\psi\rangle_{p}$ describes a single particle homogeneously delocalized within a box. The box is then partitioned into two; one of the boxes thus obtained is brought to London and the other is taken to Tokyo. When two observers, one in London and one in Tokyo, open the boxes, only one of them will find the (whole) particle.

Nevertheless, quantum mechanics tells us that, before opening, the particle was not in one of the boxes. The wavefunction describing the particle delocalized in the two boxes implies coherence between its two one-box components, i. e., these are capable of interference. It would be impossible if the particle were in one of the boxes. (Then the particle would be described by a proper mixture.) Unfortunately, the London-and-Tokyo observers are 
unable to perform an interference experiment. But in the physically isomorphic case of a two-slit experiment (cf relation (1)) this can be done (at least in an ensemble).

We easily picture the mentioned opening of the boxes in the collapse 1. To describe it in the no-collapse, relative-state approach, let us assume, for simplicity, that the observers in London and Tokyo are also in a pure state $|\phi\rangle_{o}^{L T}$. Then, before opening the boxes we have the composite state $|\phi\rangle_{o}^{L T}|\psi\rangle_{p}^{L T}$, where $|\psi\rangle_{p}^{L T}$ is the state of the particle delocalized in the London and the Tokyo boxes.

The act of opening the boxes is, in principle, a dynamical evolution that converts the last mentioned composite state into

$$
(1 / 2)^{1 / 2}\left(|\phi\rangle_{o}^{L}|\psi\rangle_{p}^{L}+|\phi\rangle_{o}^{T}|\psi\rangle_{p}^{T}\right)
$$

where the terms correspond to finding the particle in the London or the Tokyo box respectively. Note that the coherence is not destroyed; it is only elevated to the composite system. The particle is now in the proper mixture $(1 / 2)\left(|\psi\rangle_{p}^{L}\left\langle\left.\psi\right|_{p} ^{L}+\mid \psi\right\rangle_{p}^{T}\left\langle\left.\psi\right|_{p} ^{T}\right)\right.$. Needless to say that 'finding the particle in the London box', e. g., is the relative state relative to the subject state $|\phi\rangle_{o}^{L}$ in the composite state (6).

If we choose to view the state after opening the boxes via the all-encompassing decomposition (5) that we have borrowed from Sudbery, then we must join the state $|\chi\rangle_{r}$ of the rest of the world in case of opening. But we must also allow for the possibility of not opening the boxes, because a decision on part of the observers can be made or not (in a parallel world it is not made), just like it is in the case of measurement results. Besides, there may be also other world components.

Thus, instead of (6) we obtain

$$
\left.\alpha\left(|\phi\rangle_{o}^{L}|\psi\rangle_{p}^{L}+|\phi\rangle_{o}^{T}|\psi\rangle_{p}^{T}\right)\right)|\chi\rangle_{r}+\beta\left(|\phi\rangle_{o}^{L T}|\psi\rangle_{p}^{L T}|\chi\rangle_{r}^{\prime}\right)+\ldots
$$

where $|\phi\rangle_{o}^{L}$ and $|\phi\rangle_{o}^{T}$ are now the states of Sudbery's "experiencing" observers who find the particle in the London or the Tokyo box respectively, and the product of the other two wavefunctions applies to Sudbery's 'rest of the world'.

It is assumed that 'the rest of the world' is not the same when the boxes are opened and when they are not. This makes the composite state (6) the relative state with respect to the subject state $|\chi\rangle_{r}$ in the larger composite state (7), etc. (This state has to be orthogonal to all the other states of the 
rest of the world in (7).)

\section{Concluding remarks}

One might say roughly that viewing the individual-system probabilities as degrees of presence is, following the German philosopher Immanuel Kant, Ding an Sich (thing for itself), and that measuring the probabilities in ensembles in the frequentist way, when we take cognisance of the their values, is Ding für Uns (thing for us). Strictly speaking, only the very existence of a quantum system under consideration, its reality, is 'a thing for itself'. Our quantum knowledge $\left|\Phi_{n}(t)\right\rangle$ (cf relation (5)) about it, though it is a maximal quantum reality, it is relative reality, taken with respect to the state $\left|\eta_{n}\right\rangle$ of our mind, or of the measuring instrument etc. Even if we do not take (5) to be a physically meaningful decomposition of the wavefunction of everything, the lhs can be the wavefunction of another subject entity, e. g., of 'Wigner' when he considers his 'friend'. 'Relative reality' itself is a step towards the subject entity, and towards the 'thing for us'. The frequentist measurement is only the last step.

At the time of writing this article I have a fundamental dilemma. Namely, I believe that Occam's razor is a good principle for science. Applying it to the Everettian approach, we have a minimum of assumptions at the input, but a maximum of hardly acceptable final outcomes (the parallel worlds) at the output. One wonders if one should not, perhaps, apply Occam's razor partly also to the output.

If I am allowed to speculate, it seeems to me that this can be done by postulating that each system has an 'individuality beyond quantum mechanics' (not a quantum property). Then it won't branch into the different worlds and the system will remain a subsystem of the universe, not a component.

One way to achieve this might be to take suitable hidden variables. The most elaborate theory along these lines utilizes the Bohmian (nonquantum) positions of all particles (Bohm and Hiley 1993). (No two particles can be at the same place. Hence, their non-quantum position is their 'individuality beyond quantum mechanics'.)

Relation (5) is still valid, perhaps with only mathematical meaning. No more than one term (one branch) is real in the classical sense; the one into which the 'individuality beyond quantum mechanics', together with those of the rest of the classical world, 'falls' in a random way. The other terms 
are, at the instant at issue, 'unrealized former possibilities'. But they still represent quantum reality, and they may partake in forming a future state.

\section{References}

Aristotle (1980). De Interpretatione. Peripatetic Press, Grinnell, Iowa. Chapter 9.

Bell, J. S. (1990). Against 'Measurement'. Physics World, August, 33-40.

Bohm, D. and Hiley, B. J. (1993). The Undivided Universe. An Ontological Interpretation of Quantum Theory. Routledge, London.

Busch, P., Lahti, P. J. and Mittelstaedt P. (1996). The Quantum Theory of Measurement. 2nd edition. Lecture Notes in Physics. Springer, Berlin.

Busch, P. and Jaeger, G. (2010). Unsharp Quantum Reality. Foundations of Physics, 40, 1341-1367; arXiv:1005.0604v2.

Butterfield, J. (2002). Some Worlds of Quantum Theory. In Quantum Mechanics. Scientific Perspectives on Divine Action. Russell R. et al. eds. Vol. 5, 111-140. Rome: Vatican Observatory Publications, 2; arXiv: 0105052.

Byrne, P. (2010). The Many Worlds of Hugh Everett III. Oxford University Press, Oxford.

Carr, B. (2007) editor. Universe or Multiverse? Cambridge University Press, Cambridge.

Caves, C. M., Fuchs, C. A. and Schack, R. (2002). Quantum Probabilities as Bayesian Probabilities. Physical Review A, 65, 022305 (6 pages); arXiv:quant-ph/0106133v2.

D'Espagnat, B. (1976). Conceptual Foundations of Quantum Mechanics. Second edition. W. A. Benjamin, Reading, Massachusetts, subsection 7.2

De Witt, B. and Graham, N. editors. (1973). The Many-Worlds Interpretation of Quantum Mechanics. Princeton University Press, Princeton.

De Witt, B. S. (2004). The Everett Interpretation of Quantum Mechanics. In Science and Ultimate Reality. Editors J. D. Barrow et al. Cambridge University Press, Cambridge. 167-198.

Everett, H. (1957). "Relative State" Formulation of Quantum Mechanics. Reviews of Modern Physics, 29, 454-462. Reprinted in De Witt and Graham (1973), 141-150.

Everett, H. (1973). The Theory of the Universal Wave Function.

In De Witt B. and Graham N. (1973), 1-140.

Ghirardi, G.-C. (2008). Reconsidering Mermin's "In Praise of Measurement". 
arXiv:0806.0647

Gleason, A. M. (1957). Measures on the Closed Subspaces of a Hilbert Space. Journal of Mathematics and Mechanics, 6, 885-893.

Gottfried, K. (1991). Does Quantum Mechanics Carry the Seeds of its Own Destruction? Physics World, October, 34-40.

Heisenberg, W. (1958). Physics and Philosophy. Harper and Brothers Publication, New York.

Heisenberg, W. (1959). The Discovery of Planck and the Philosophic Problems of Atomic Physics. (In German) Universitas, 14, 135-153.

Herbut, F. (1992). Quantum Interference Viewed in the Framework of Probability Theory. American Journal of Physics, 60, 146-150.

Herbut, F. and Vujičić M. (1997). First-Quantisation Quantum-Mechanical Insight into the Hong-Ou-Mandel Two-Photon Interferometer with Polarizers and its Role as a Quantum Eraser. Physical Review, A 56, 1-5.

Herbut, F. (2004). On Compatibility and Improvement of Different Quantum State Assignments. Journal of Physics A: Mathematical and General, 37, 1-8; arXiv:quant-ph/04051.

Herbut, F. (2005a). A Quantum Measure of Coherence and Incompatibility. Journal of Physics, A 38, 1046-1064.

Herbut, F. (2005b). Can We Believe in a Purely Unitary Quantum Dynamics? ArXiv:quant-ph/0507218

Herbut, F. (2005c). Mutual Information of Bipartite States and Quantum Discord in Terms of Coherence Information. International Journal of Quantum Information, 3, 691-728.

Herbut, F.(2007). Quantum Probability Law from 'Environment-Assisted Invariance' in Terms of Pure-State Twin Unitaries. Journal of Physics A: Mathematical and Theoretical, 40, 5949-5971; arXiv: quant-ph/0611220.

Herbut, F. (2008a). On EPR-type Entanglement in the Experiments of Scully et al. I. The Micromaser Case and Delayed-Choice Quantum Erasure. Foundation of Physics, 38, 1046-1064; arXiv: quant-ph/0808.3176 .

Herbut, F. (2008b). Quantum Correlations in Multipartite States. Study Based on the Wootters-Mermin Theorem. arXiv:0811.3674

Herbut, F. (2010a). On EPR-type Entanglement in the Experiments of Scully et al. II. Insight in the Real Random Delayed-Choice Erasure Experiment. Foundations of Physics, 40, 301-312; arXiv: quant-ph/0808.3177.

Herbut, F. (2010b). A Theory of Quantum Preparation. arXiv:1005.1348

Herbut, F. (2011). How Can the No-Collapse and the Collapse Interpretations of Quantum Mechanics Give the Same Description? arXiv:1108.4175 
Hulpke et al. (2006). Hulpke, F., Poulsen, U. F., Sanpera, A., Sen(De), A., Sen, U., and Lewenstein, M. Unitarity as Preservation of Entropy and Entanglement in Quantum Systems. Foundations of Physics, 36, 477499.

Joos, E. et al. editors (2003). Decoherence and the Appearance of a Classical World in Quantum Theory. Springer, Berlin.

Kim, Y.-H., Yu, R., Kulik, S. P., Shih, Y. and Scully, M. O. (2000). Delayed "Choice" Quantum Eraser. Physical Review Letters, 84, 1-5.

Lukasiewicz, J. (1970). Logical Foundations of Probability Theory. In $\mathrm{Se}$ lected Works edited by L. Borkowski. North-Holland, Amsterdam, 1663. (First published in Cracow 1913).

Mermin, N. D. (1998). What is Quantum Mechanics Trying to Tell us? American Journal of Physics, 66, 753-767; arXiv: quant-ph/9801057v2.

Mermin, N. D. (2006). In Praise of Measurement. Quantum Information Processing 5, 239-260; ArXiv:quant-ph/0612216

Norsen, T. (2005). Einsteins Boxes. American Journal of Physics, 73, 164176.

Pauli, W. (1994). Probability and Physics. In Writings on Physics and Philosophy. Springer-Verlag, New York 43-48.

Peierls, R. E. (1991). In Defense of "Measurement". Physics World, January, 19-20.

Petersen, A. (1963). The Philosophy of Niels Bohr. The Bulletin of the Atomic Scientists. September, p. 8.

Popper, K. R. (1982). Quantum Theory and the Schism in Physics. Hutchinson, London.

Popper, K. R. (1983). Realism and the Aim of Science. Hutchinson, London. Prior, A. (1967). Past, Present and Future. Clarendon Press, Oxford.

Reichenbach, H. (1949). The Theory of Probability. University of California Press.

Reichenbach, H. (1965). Philosophic Foundations of Quantum Mechanics. University of California Press, Berkeley. (First published in 1944).

Saunders, S. (1998). Time, Quantum Mechanics, and Probability. Synthese, 114, 373-404; quant-ph/0111047.114.

Saunders, S. et al. editors (2010). Many Worlds? Oxford University Press, Oxford.

Schröddinger, E. (1935a). The Present Situation in Quantum Mechanics. A Translation of Schrödinger's "Cat Paradox" Paper. In Wheeler and Zurek (1983) 152-167. 
Schrödinger, E. (1935b). Discussion of Probability Relations Between Separated Systems. Proc. Cambridge Phil. Soc. 31, 555-563.

Schrödinger, E. (1936). Probability Relations Between Separated Systems. Proc. Cambridge Phil. Soc. 32, 446-452.

Selleri, F. and van der Merwe, A. (1991). Karl Popper at Ninety: Highlights of a Lifelong Intellectual Quest. Foundations of Physics, 21, 1375-1386.

Shimony, A. (1993). Search for a Naturalistic World View. Vol. II. Cambridge University Press, Cambridge.

Stapp, H.P. (1972). The Copenhagen Interpretation. American Journal of Physics, 40, 1098-1116.

Suárez, M. (2004). On Quantum Propensities; 2 Arguments Revisited. Erkenntniss, 61, 1-6.

Suárez, M. (2007). Quantum Propensities. Studies in History and Philosophy of Modern Physics, 38, 418-438.

Sudbery, A. (2011). Philosophical Lessons of Entanglement. Talk given at 75 Years of Quantum Entanglement Kolkata, India. 10 January 2011. arXiv:1103.4318v1.

Von Neumann, J. (1955) Mathematical Foundations of Quantum Mechanics. Princeton University Press, Princeton.

Wheeler, J. A. (1957). Assessment of Everett's "Relative State" Formulation of Quantum Theory. Reviews of Modern Physics, 29, 463-465.

Wheeler, J. A. (1977). Include the Observer in the Wave Function? In Quantum Mechanics, a Half Century Later 1-18. Editors J. L. Lopes and M. Paty. D. Reidel Publishing Company, Dordrecht, Holland.

Wheeler, J. A. and Zurek, W. H. editors (1983). Quantum Theory and Measurement. Princeton University Press. Princeton.

Whitaker, M. A. B. (2008). Foundations of Physics, 38, 436-447.

Wigner, E. (1961). Remarks on the Mind-Body Question. In Wheeler and Zurek (1983), 168-181.

Wigner, E. P. (1973). Epistemological Perspective on Quantum Theory. In Contemporary Research in the Foundations and the Philosophy of Quantum Theory. Editor C. A. Hooker. Reidel, Dordrecht, Holland, 369-385.

Zeh, H. D. (1970). On the Interpretation of Measurement in Quantum Theory. Foundations of Physics, 1, 69-76. 